\title{
Impaired Poststenotic Aortic Pulsatility After Hemodynamically Ideal Coarctation Repair in Children
}

\author{
J.-P. Pfammatter, ${ }^{1}$ P. Berdat, ${ }^{2}$ T. Carrel $^{2}$ \\ ${ }^{1}$ Department of Pediatric Cardiology, University Hospital Berne, Berne, CH 3010, Switzerland \\ ${ }^{2}$ Department of Cardiovascular surgery, University Hospital Berne, Berne, CH 3010, Switzerland
}

\begin{abstract}
Using echocardiographic quantification of aortic pulsatility distal to the site of the surgical anastomosis, we evaluated whether the preoperatively impaired poststenotic aortic pulsatility returned to normal after repair of coarctation with a hemodynamically ideal result. Patients who underwent repair of aortic coarctation without residual obstruction were compared to a matched group of normal children. A standardized M-mode echocardiographic evaluation of the aorta at the diaphragmatic level was performed for all patients. Measurements consisted of maximum and minimum aortic diameters, time intervals, and a calculated pulsatility index. Compared to normal children $(n=19), 20$ children with operated coarctation and with a hemodynamically ideal result showed a significantly smaller increase in aortic diameter in systole (mean of $29 \pm 7 \%$ in patients versus $37 \pm 7 \%$ in normals; $p<0.01$ ). In contrast to patients with coarctation in whom the maximum aortic distension is reached much later during the cardiac cycle, hemodynamically normalized, operated patients in our study had no such delay (maximum aortic pulsation at $28 \%$ of cardiac cycle time compared to $27 \%$ in normals; $p=$ not significant). The pulsatility index of the poststenotic aorta was clearly lower in operated children (mean, $130 \pm 50 \% / \mathrm{sec}$ ) compared to a normal mean value of $202 \pm 33 \% / \mathrm{sec}$ but was still significantly higher than that in patients with unoperated coarctation, who showed a low mean value of $51 \pm 24 \% / \sec (p<0.01)$. After correction of aortic coarctation with a hemodynamically ideal result, the pulsatility of the poststenotic aorta, severely impaired prior to repair, did not return to normal during the observation period in the patients studied.
\end{abstract}

Correspondence to: J.-P. Pfammatter, email: jean-pierre.pfammatter@insel.ch
Keywords: Aortic coarctation - Coarctation repair

In children with coarctation, the discrete aortic obstruction leads to a pressure drop and loss of the pulse wave pressure distal to the stenosis, manifesting with the typical clinical sign of absent pulses in the lower limbs. The characteristics of the pulsatility of the poststenotic (thoracic) aorta, determined using a pulsatility index derived from a simple M-mode echocardiographic evaluation, have been reported by our group [5]. It was shown that the pulsatility index of the poststenotic aorta allowed for a clear discrimination between patients with unoperated coarctation and normals.

In the current study, we prospectively investigated whether an ideal hemodynamic result after surgical correction of coarctation would lead to normalization of the pulsatility characteristics of the poststenotic aorta.

\section{Methods}

\section{Patients}

All consecutive patients seen in our outpatient clinic for routine follow-up evaluation after repair of coarctation were considered for this study. The study period was 2 years, ending in March 2001. Inclusion criteria for the group of operated patients without recoarctation were coarctation repair by resection and (extended) endto-end anastomosis, normal left ventricular function on echocardiography with a fractional shortening $>30 \%$, normal aortic arch by two-dimensional echo imaging, corrected Doppler gradient in the descending aorta $<20 \mathrm{mmHg}$, normal femoral pulses, normal right arm blood pressure for age [1] and no blood pressure gradient between upper and lower limbs (i.e., blood pressure in lower limbs equal or higher than that in upper limbs).

Exclusion citeria were uncooperative patient with poor image quality (no sedation used), patients on cardiovascular drugs, and associated congenital heart disease except for a bicuspid aortic valve with transvalvar Doppler gradient $<20 \mathrm{mmHg}$. 

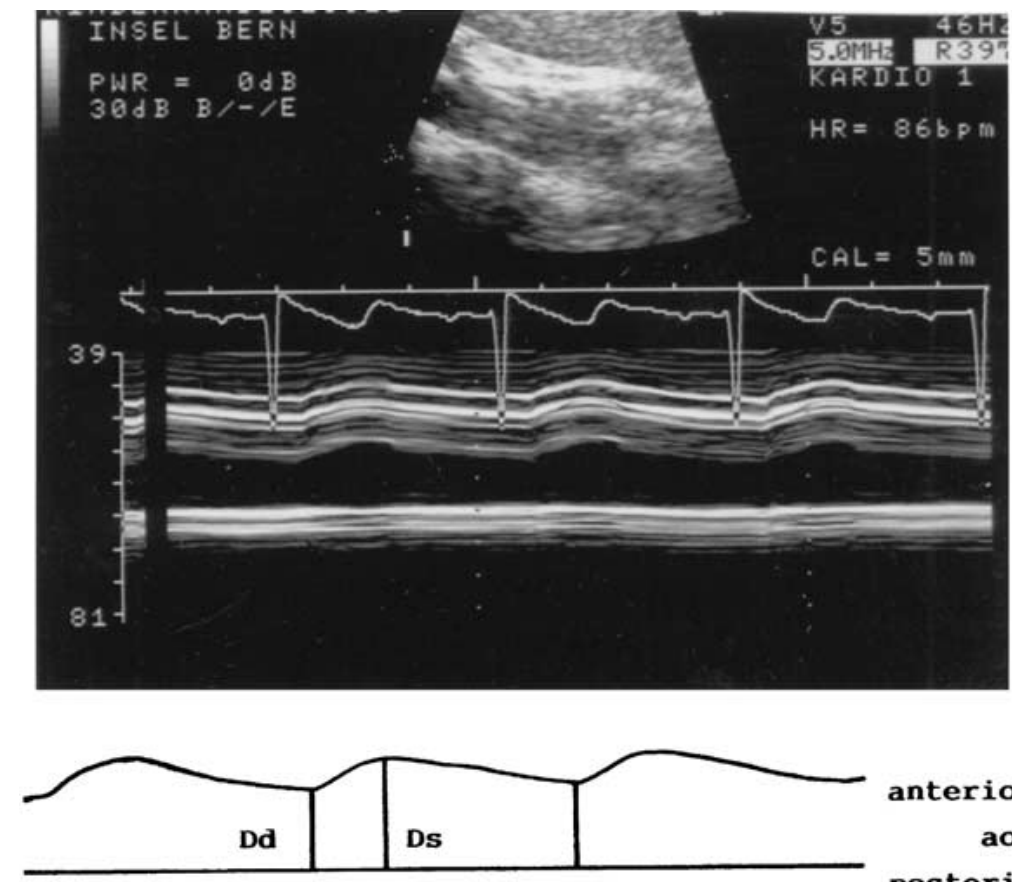

anterior

aortic wall posterior
Fig. 1. (Top) Example of an Mmode echocardiographic image of the thoracic aorta at the diaphragmatic level: shown is the aortic pulsatility in a normal child. (Bottom) A schematic representation of the sites of measures taken for the inner aortic diameters, $D d$, minimum inner diameter; $D s$, maximum inner diameter; $t_{u}$, the upstroke time - the interval between Dd and Ds; $t_{R R}$, cardiac cycle time.
The normal population was selected from children evaluated for a heart murmur in the outpatient clinic. They were matched for age and sex and underwent the same echocardiographic protocol as the patient, and they had blood pressure measurements as described for patients. The study was approved by the institutional ethics committee.

\section{Blood Pressure Measurements}

Blood pressure measurements were made using the oscillometric method (Dinamap, Critikon) using an appropriately sized cuff [1]. Measurements were made on all four limbs and all values were taken as the mean of three consecutive measurements. Only systolic blood pressure measurements were evaluated.

\section{Echocardiographic Measurements}

All patients had a complete two-dimensional and Doppler echocardiographic evaluation with visualization of the site of operation from the suprasternal notch. Maximum blood flow velocities were measured in the axis of the ascending and descending aorta, and the maximum Doppler gradient in the descending aorta was corrected according to the original Bernoulli formula for the velocity in the ascending aorta.

The technique of M-mode echocardiographic evaluation of aortic pulsatility has been described previously [5]. Briefly, the sector array transducer $(5 \mathrm{MHz}$ in all patients) of the echo ma- chine (Acuson 128 XP 10, Acuson, Mountain View, CA, USA) was kept longitudinally along the axis of the thoracic aorta at the level of the diaphragm. The picture obtained was magnified using the resolution box function. A simultaneous one-lead electrocardiograph (ECG) was displayed on the echo monitor at a speed of $50 \mathrm{~mm} / \mathrm{sec}$. As shown in Fig. 1, the aortic inner diameters were measured at end diastole $\left(D_{d}\right)$ and maximum systole $\left(D_{s}\right)$ as the distance between the trailing edge of the anterior aortic wall and the leading edge of the posterior aortic wall. The time interval between these two measures $\left(t_{u}\right)$ was measured and indexed to the duration of the whole cardiac cycle ( $\%$ of cardiac cycle time). From these measurements, we calculated the percentage increase in the aortic inner diameter (expressed as $\%$ of $D_{d}$ ) as well as the pulsatility index calculated from the maximum increase in inner diameter indexed to the time $(\% / \mathrm{sec})$ as age- and weight-independent indexes.

For measurements and calculations, the frozen echocardiographic image was blinded and coded on a printout (Mitsubishi K70S video copy processor). All values were obtained as the mean of three consecutive cardiac cycles measured. The calculation of the intraobserver variability has been described in detail [5]. used.

All patients were in the supine position, and no sedation was

\section{Statistical Analysis}

Descriptive data are shown as median with the range or mean with 1 standard deviation where appropriate. Comparison between 
Table 1. Comparison of echocardiographic measurements in patient groups

\begin{tabular}{|c|c|c|c|}
\hline Parameter & Coarctation patients & Patients with repaired coarctation & Normals \\
\hline No. of patients & 27 & 20 & 19 \\
\hline Median age (years) & 4.2 & 5.9 & 6.1 \\
\hline LVFS (mean, \%) & 39 & 35 & 37 \\
\hline Minimum aortic diameter (mm) & $7.1 \pm 1.0$ & $8.5 \pm 2.8$ & $8.2 \pm 2.0$ \\
\hline Maximum aortic diameter (mm) & $8.0 \pm 1.5$ & $10.4 \pm 2.4$ & $10.9 \pm 2.0$ \\
\hline Increase in aortic diameter $(\%)$ & $13 \pm 6$ & $29 \pm 7$ & $37 \pm 7$ \\
\hline Time of upstroke (msec) & $251 \pm 53$ & $183 \pm 60$ & $185 \pm 29$ \\
\hline Time of upstroke (\% of RR) & $39 \pm 5$ & $28 \pm 5$ & $27 \pm 5$ \\
\hline Pulsatility index $(\% / \mathrm{sec})$ & $51 \pm 24$ & $130 \pm 50$ & $202 \pm 33$ \\
\hline
\end{tabular}

LVFS, left ventricular fractional shortening; RR, cardiac cycle time

groups was made with analysis of variance using commercially available software (Statview). A $p$ value-of $<0.05$ was considered statistically significant.

\section{Results}

\section{Patient Characteristics}

A group of 20 patients with operated coarctation (3 girls and 17 boys) and with a hemodynamically ideal postoperative result were included. Their age at the time of the present evaluation varied between 2.2 and 16 years (medians, 5.9), and their weight varied between 13 and $57 \mathrm{~kg}$ (median, 21). The age at the time of coarctation repair was 3 weeks to 4.7 years (median, 1.6 years). The time from operation to the current evaluation was 1.0-10.8 years (median, 5.2). Surgery consisted of coarctation resection with a direct end-to-end anastomosis in all children, with an anastomosis extended into the aortic arch in 7 patients with aortic arch hypoplasia. Associated bicuspid aortic valves with transvalvar Doppler gradients between 7 and $17 \mathrm{mmHg}$ were observed in 6 children.

The normal population consisted of 19 healthy children ( 3 girls and 16 boys) with an age between 1.4 and 13.4 years (median, 6.1) and a weight between 10 and $48 \mathrm{~kg}$ (median, 23).

For comparison, data of 27 patients with unrepaired coarctation were also analyzed. These data were derived partially from our initial study group [5] and extended with new patients observed in the meantime $(n=8)$. The age of these patients with coarctation varied between 0.2 and 12.1 years (median, 4.2).

\section{Hemodynamic Data}

In all patients with repaired coarctation, systolic right arm blood pressure was in the normotensive range, with values between the 90th and 95th percentile in 10 children, between the 75 th and 90 th percentile in 8
$(\% / \mathrm{sec})$

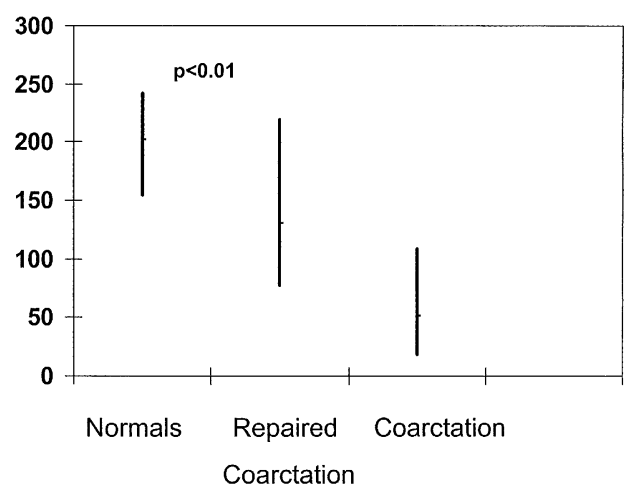

Fig. 2. Comparison of the pulsatility index (median and range) poststenotic thoracic aorta between the patient groups.

children, and below the 75th. percentile in the remaining 2 children. There was no blood pressure gradient between upper and lower limbs in any patient.

The maximum Doppler gradient in the descending aorta varied between 6 and $20 \mathrm{mmHg}$ (median, 16), with no diastolic gradient in any of the patients.

\section{Echocardiographic Evaluation of Aortic Pulsatility}

The echocardiographic measurements in the different patient groups are shown in Table 1. The minimum inner diameter of the thoracic aorta at the point of measurements was the same in patients with repaired coarctation and normal children. In the group of patients with an ideal result of coarctation repair, the increase in thoracic aortic diameter from diastole to the maximum in systole was significantly lower than that in the group of normal children $(p<$ 0.01 ), but for both groups it was significantly higher than that in patients with unoperated coarctation $(p$ $<0.001$ ). Time intervals for the aortic distension did not differ between normals and operated children. 
There was also no difference when the time to maximum aortic elongation was expressed in relation to the duration of the cardiac cycle time. However, these values differed significantly for normals and operated children when compared to those of unoperated patients $(p<0.01)$.

When calculating the pulsatility index of the poststenotic thoracic aorta, there was a significant difference between normal children and patients after successful coarctation repair with lower values for operated coarctation patients $(p<0.01)$. Both these groups significantly differed from patients with unrepaired coarctation, who had a pulsatility index lower than those of normals and operated patients ( $p$ $<0.01$; Fig. 2).

\section{Discussion}

The main finding of this study was that aortic pulsatility distal to the site of obstruction did not completely return to normal after surgical correction with an ideal hemodynamic result. Compared to a matched group of normal children, there is a clear statistical difference in poststenotic aortic pulsatility following successful surgery, although there was a wide overlap with normal values. This was in contrast to patients with unoperated coarctation, in whom poststenotic aortic pulsatility showed a clear discrimination without overlap with normal values [5].

Several explanations are possible for the observation that an ideal surgical result did not lead to normalization of aortic pulsatile behavior. Ventricular function may be one focus because it has been reported that even in long-term follow-up after coarctation repair without residual stenosis, altered systolic and diastolic left ventricular function persisted [4]. It remains unclear whether ventricular functional alteration is not by itself an expression of altered arterial vessel physiology in such patients. The protocol used in our study did not allow for exclusion of subtle alterations in ventricular function.

Based on the frequent observation of arterial hypertension in patients with successfully repaired coarctation, many investigations have concentrated on the function of arteries in patients with coarctation. Using echocardiographic methods similar to those used in our evaluation, aortic and arterial compliance have been investigated in the prestenotic and poststenotic arterial vascular bed. An impaired dilatory capacity of the precoarctation arterial vascular bed has been found in young patients without residual stenosis [3]. These findings were independent of the age at surgical repair, consistent with our findings. Interestingly, in that same study [3] the large vessel dysfunction was not found in the femoral arteries in the patients tested and thus was confined to the precoarcation arterial bed. Similar findings were found using transesophageal echocardiography, where impairement of the aortic elastic properties in the precoarctation aorta was more pronounced than in the thoracic aorta [2]. In addition, that study showed an effect of age at the time of operation; a lower distensibility index of the aorta correlated with older age at time of repair. Aortic distensibility and compliance were also assessed in patients who underwent balloon dilatation as an alternative to surgical resection of coarctation. In these patients, it was found that arterial dysfunction mainly occurred in the prestenotic aortic segment [7].

The impaired pulsatility of the thoracic aorta following surgical repair might also be explained by the fact that the circumferential scar at the operation site disturbed the propagation of the pulse wave. However, it has been experimentally shown in an animal model that even implantation of a stent at the site of coarctation does not alter pressure and flow characteristics in the descending aorta [6]. Mild residual stenosis not detected with the clinical methods used might be another possible explanation for impaired aortic pulsatility.

Clinical implications include the following: structural alterations in the aortic wall even outside the distinct narrowed segment have been postulated [7], implicating that coarctation is more than just a regional stenosis. Frequently observed abnormalities in blood pressure, even in successfully repaired patients, would be a strong indicator for such a hypothesis. Many more factors (e.g., humoral) should be taken into account to adequately address this complex matter. Covering just one mechanistic aspect of arterial physiology in coarctation patients, we started with the initial observation of a very characteristic pattern of poststenotic aortic pulsatility in coarctation [5] and extended this diagnostic application to postoperative patients.

\section{References}

1. Anonymous (1996) Update on the 1987 task force report on high blood pressure in children and adolescents: a working group report from the National High Blood Pressure Education Program. Pediatrics 98:649-658

2. Brili S, Dernellis J, Aggeli C, et al. (1998) Aortic elastic properties in patients with repaired coarctation of aorta. Am J Cardiol 82:1140-1143

3. Gardiner HM, Celermajer DS, Sorensen KE, et al. (1994) Arterial reactivity is significantly impaired in normotensive young adults after successful repair of aortic coarctation in childhood. Circulation 89:1745-1750 
4. Moskowitz WB, Schieken RM, Mosteller M, Bossano R (1990) Altered systolic and diastolic function in children after successful repair of coarctation of the aorta. Am Heart J 120:103109

5. Pfammatter JP, Stocker FP (1999) Quantitative echocardiographic characterization of abdominal aortic pulsatility in children with coarctation. Pediatr Res 46:126-130
6. Pikhala J, Thyagarajan GK, Taylor GP, Nykanen D, Benson LN (2001) The effect of implantation of aortic stents on compliance and blood flow. An experimental study in pigs. Cardiol Young 11:173-181

7. Xu J, Shiota T, Omoto R, et al. (1997) Intravascular ultrasound and assessment of regional aortic wall stiffness, distensibility and compliance in patients with coarctation of the aorta. Am Heart $J$ 134:93-98 